\title{
Cambio y psicoterapia: la perspectiva del niño*
}

\section{Change and Psychotherapy: The Child's Perspective}

Malenka Areas*
ORCID: 0000-0002-4286-9703
Julieta Olivera
ORCID: 0000-0001-8187-0363

Universidad de Belgrano, Argentina

Andrés Roussos

ORCID: 0000-0002-5138-9664

Universidad de Belgrano y Conicet,

Argentina

Recibido: 22 de febrero de 2020

Revisado: 7 de marzo de 2020

Aceptado: 22 de abril de 2020

\section{Resumen}

La investigación a partir de la perspectiva del paciente ha brindado información importante acerca de cómo la terapia es percibida, ayudando a los terapeutas a entender algunas reacciones desde los pacientes. Debido a las pocas investigaciones de este tipo en la población infantil, la terapia con niños no ha sido beneficiada por este movimiento. El presente trabajo es de carácter empírico con un enfoque de análisis cualitativo. En este estudio se exploraron las percepciones de los niños sobre distintos elementos del proceso psicoterapéutico. Para esto, se realizaron entrevistas semiestructuradas a 10 niños entre 6 y 12 años que se encontraban realizando tratamiento psicoterapéutico, o lo habían realizado en el último año. Durante las entrevistas se indagó sobre la "relación terapéutica”, las “intervenciones del terapeuta", la "valoración de la terapia", las "nociones sobre la terapia", las "características de la terapia" y el "cambio percibido". Se analizaron las entrevistas bajo una metodología cualitativa consensual (CQR). Los resultados indican que los niños percibieron cambios vinculados a su propia conducta, emociones y relaciones interpersonales y pudieron relacionarlos con sus terapeutas y la alianza establecida con ellos.

Palabras clave: Cambio, Perspectiva del paciente, Investigación cualitativa, Psicoterapia de niños, Relación terapéutica en niños.

\footnotetext{
* Artículo de investigación. Este trabajo es producto del proyecto de investigación Conicet y la Universidad de Belgrano. Citar como: Areas, M., Olivera, J. y Roussos, A. (2020). Cambio y psicoterapia: la perspectiva del niño. Diversitas: Perspectivas en Psicología, 16(2), 245-257. https://doi.org/10.15332/22563067.5553

** Correspondencia: Malenka Areas, Universidad de Belgrano. Correo electrónico: malenka.areas@gmail.com Julieta Olivera, Universidad de Belgrano. Correo electrónico: julieta.olivera@comunidad.ub.edu.ar Andrés Roussos, Universidad de Belgrano y Conicet. Correo electrónico: andres.roussos@comunidad.ub.edu.ar
} 


\begin{abstract}
Research from the patients' perspective has provided valuable information about how the psychotherapy process is perceived, helping therapists to understand some of the patient's reactions. Due to the limited research of this type in the children population, therapy with children has not benefited from this movement. The present study is an empirical work framed in a qualitative analysis approach, in which we inquired about children's perceptions about different elements related to the psychotherapeutic process. For this purpose, we conducted semi-structured interviews with 10 children between 6 and 12 years old, who were undergoing a psychotherapeutic treatment or had done it in the last year. During the interviews, we inquired about elements such as "therapeutic relationship," "therapist interventions," "therapy assessments," "notions about therapy," "therapy characteristics," and "perceived change." The interviews were analyzed using a consensual qualitative methodology (CQR). The results indicate that children were able to perceive changes concerning their behavior, emotions, and interpersonal relationships and could link these changes with their therapists and the therapeutic alliance established with them.
\end{abstract}

Keywords: change, patient perspective, qualitative research, child psychotherapy, therapeutic relationship in children.

\section{Introducción}

Las personas realizan psicoterapia por diversas razones, ya sea para generar un cambio en un conjunto de síntomas o buscar mejorar la calidad de vida. Es así como la promoción del cambio se establece como uno de los objetivos principales del tratamiento psicoterapéutico en la actualidad (Fernández Álvarez, 2011). Roussos (2013) considera que el cambio psicoterapéutico está relacionado a un crecimiento que surge a lo largo de un tratamiento. Como plantean Laurenceau, Hayes y Feldman (2007) el estudio y la medición del cambio desde la perspectiva del paciente nos da la oportunidad de observar el estado de evolución de los pacientes durante y posteriormente a una intervención o tratamiento.

Las intervenciones utilizadas en psicoterapia en niños y en adultos suelen ser distintas. Algunas de las más conocidas en la clínica con adultos de terapia cognitiva-conductual (TCC) son la psicoeducación, la implementación de registros organizados de pensamientos, técnicas de cuestionamiento guiado inspiradas en la mayéutica socrática y la realización de tareas inter-sesión (Bunge, Gomar y Mandil,
2009). En el trabajo con niños, estas intervenciones pueden encontrarse con limitaciones debido a la escasa motivación para realizar el tratamiento, el estadio del desarrollo intelectual, y la capacidad de comunicación que presentan los pequeños. Por lo tanto, las intervenciones que se utilizan en esta etapa están sujetas al momento del desarrollo de cada niño, lo que muchas veces implica que sean más "simples", orientadas a cuestiones concretas (Bunge et. al., 2009).

Teniendo en cuenta estas condiciones, distintos autores mencionan que con los niños "las palabras no son suficientes", por lo cual se le otorga un valor a la inclusión de la acción y la experiencia en el trabajo terapéutico (Stern, 2002). Es por esto que la inclusión de distintas modalidades como el juego y los dibujos, tanto para recibir como para expresar información, se utilizan como vehículo de gran parte de las intervenciones (Stern, 2002). En el caso del juego, Landreth (2012) explica que el mundo de los niños es de realidades concretas, y sus experiencias suelen comunicarse a través del juego mismo, que es su medio natural de comunicación. Por otro lado, el dibujo, puede ser para los niños una forma 
de explorar tanto, su mundo interno como externo, y de descubrir habilidades. A través del uso de los dibujos, los niños pueden comunicar ideas complejas, y mensajes que a través de su vocabulario sería difícil expresar (Malchiodi, 2003). Desde una perspectiva similar, Drake y Winner (2012) plantean que el dibujo se considera una de las actividades de placer que los niños suelen utilizar para contrarrestar estados de ánimo negativos. Teniendo en cuenta la forma en la que los niños se ven involucrados por esta actividad, las autoras sostienen que muchas veces esto los puede ayudar con la regulación de sus emociones (Drake y Winner, 2012).

Entendiendo que los tratamientos con niños difieren de los tratamientos con adultos, es imprescindible que se realicen investigaciones específicas que aporten información a los psicoterapeutas que trabajan con estos pacientes. Sempik, Ward y Darker (2008) señalan que uno de cada diez jóvenes, de entre 5 a 16 años es diagnosticado con alguna condición de salud mental (Sempik, Ward, y Darker, 2008). A pesar de esta alta incidencia, la investigación en salud mental dirigida a niños es una de las de menor presupuesto, y por lo tanto de menor producción (Midgley et al., 2018). Esto conduce a que los abordajes dirigidos a niños tengan menor fundamento empírico en comparación a investigaciones sobre abordaje en adultos.

\section{Investigación en Psicoterapia de niños}

El desarrollo de investigaciones sobre abordajes y sus efectos en niños resulta un desafío fundamental a nivel internacional (Midgley et al., 2018). Kazdin y Durbin (2012) resaltan la importancia de entender cuál es el motivo que perciben los niños como causa de su asistencia al tratamiento, dado que los niños no suelen ir por su propia voluntad en el comienzo del tratamiento y los objetivos pueden no estar claros para ellos. También destacan la importancia de explorar las actitudes que los niños toman en relación al cambio, el establecimiento de metas y tareas de la terapia (Kazdin y Durbin, 2012).

La presente investigación se refiere a la experiencia de la psicoterapia por parte de los pacientes, que en este caso son niños entre 6 y 12 años. Como antecedentes, uno de los estudios que abordó esta temática fue el de Diamond y Lev-Wiesel (2016) en el cual se entrevistaron adultos que habían participado de un tipo de terapia conocido como "Terapia de Arte Expresiva” (en inglés, Expressive Art Therapy), cuando eran niños. Algunos de los resultados de esta investigación incluyen la percepción de una falta de objetivos al comienzo del tratamiento y la vinculación de la terapia con la posibilidad de que se generara mayor bienestar cotidiano. Los participantes de este estudio también resaltaron que la motivación principal para asistir al tratamiento se vinculaba con la diversión y el juego.

También Midgley y colaboradores (2006) indagaron con adultos que habían realizado tratamientos psicoanalíticos durante su infancia. En este caso analizó cómo éstos percibían que la terapia había afectado sus experiencias posteriores. Inicialmente encontró que muchos tuvieron la dificultad de reflexionar sobre cómo hubiese sido su vida si no hubieran acudido al análisis. Sin embargo, la mayoría de los participantes destacaron que el simple hecho de "poder hablar" había sido valioso, y que había tenido consecuencias positivas.

Estas investigaciones, si bien presentan como meta explorar la experiencia de psicoterapia de niños, se realizaron retrospectivamente cuando éstos ya eran adultos, lo que presupone un sesgo generado por el gran distanciamiento entre la experiencia y la administración de las entrevistas.

La estrategia relacionada con indagar directamente sobre los niños y adolescentes, en lugar de adjudicarles una noción desde el punto de vista de los adultos que están a cargo, ya sea de los niños o de sus intervenciones, ha sido utilizada también por fuera de la clínica con objetivos que pueden ser asimilables al fenómeno clínico. Por ejemplo, en un trabajo orientado a establecer cómo perciben los adolescentes su presente y su futuro uno de los datos fundamentales fue la divergencia existente entre la percepción que los adolescentes tenían de sí mismos en relación con la evaluación de los adultos (Molina et al., 2017). Considerar dichos puntos de vista brinda la oportunidad de generar construcciones alternativas a la hora de la comunicación y la influencia que se puede promover desde los adultos hacia los más jóvenes (Molina et al., 2017). 
En este mismo sentido se encuentra el trabajo realizado por Castaño, Posada y Agudelo (2019) en donde se indagaron las necesidades de adolescentes en cuanto a recepción de políticas públicas vinculadas a la educación sexual en Colombia. En este trabajo encontraron que justamente la carencia de conocimiento sobre la opinión de los jóvenes era un factor que obstaculizaba el empoderamiento y la utilidad de dichos programas.

A pesar de la importancia de dichos artículos, puede observarse que los mismos se encuentran centrados en población adolescente y en temáticas de carácter social, lo cual da cuenta de la dificultad con la que nos encontramos al trabajar los psicoterapeutas con niños en el área de la psicología clínica. Teniendo en cuenta la escasez de información sobre la perspectiva que tienen los niños sobre los tratamientos que se encuentran realizando (o finalizaron recientemente) consideramos pertinente interrogarnos: ¿Qué cambios perciben los niños como resultado de su psicoterapia? ¿Cómo es la concepción que poseen de "la psicoterapia"? ¿Qué aspectos del tratamiento destacan como positivos (o negativos) ?, entre otras.

De esta manera, el objetivo de esta investigación consiste en indagar la autopercepción de los niños sobre su experiencia en psicoterapia, considerando el cambio como un elemento central. Para esto, se exploraron las nociones que los niños poseían sobre la psicoterapia en general, los factores valorados de su propio proceso psicoterapéutico y con relación a su terapeuta, los cambios percibidos a partir del mismo y las construcciones en torno a las actividades realizadas durante el tratamiento. La presente investigación, de carácter exploratoriodescriptivo, busca realizar un aporte a la evidencia existente sobre la temática de la perspectiva del paciente en niños.

\section{Métodos}

\section{Participantes}

Se utilizó un muestreo de bola de nieve (Cantoni Rabolini, 2009) para convocar a los participantes.
Se apuntó a conseguir heterogeneidad de edades dentro del marco poblacional para dar cuenta de la diversidad del objeto de estudio.

Los criterios de inclusión de la muestra requirieron que el participante tuviera entre 6 y 12 años, diesen su asentimiento de participación, la autorización de los padres y se encontrasen realizando un tratamiento psicoterapéutico o hubieran realizado un tratamiento psicoterapéutico dentro del último año. Se excluyeron de la muestra aquellos niños con indicios de criterios diagnósticos de trastorno mental grave y con dificultades en la comprensión de consignas. Si bien no se aplicó ninguna evaluación psicopatológica para establecer diagnósticos, se tuvo en cuenta la capacidad de los niños para poder comprender las preguntas de las entrevistas y poder elaborar una respuesta.

A lo largo de la administración de entrevistas se convocaron 17 niños. Cinco no llegaron a participar de la entrevista ya sea por no responder a la invitación (2) o porque no se pudo concretar un día y horario para hacerla (3). Por otro lado, dos niños que fueron entrevistados presentaron dificultades en la comprensión tanto de las preguntas como de las respuestas, por lo cual sus materiales no fueron incluidos en el presente estudio.

La muestra final se compuso de 10 niños que cumplían con las condiciones de inclusión. La mitad de los niños ya habían finalizado su tratamiento, y la otra mitad aún se encontraban en proceso del mismo. Todos los niños eran de nacionalidad argentina, vivían en el Área Metropolitana de Buenos Aires y se encontraban realizando sus estudios primarios básicos. Ocho participantes eran varones y sus edades se comprendían entre los 6 y 12 años cumplidos $(M=8.6 ; D E=1.8)$. Tres participantes habían realizado anteriormente otro tratamiento psicoterapéutico. Cinco de ellos realizaron tratamiento de forma privada, los cinco restantes tuvieron algún tipo de financiación del sistema de salud del estado o su medicina prepaga. Si bien se preguntó a los padres si conocían el marco teórico del abordaje, ninguno de ellos supo la orientación del terapeuta (Tabla 1). 
Tabla 1.

Datos Composición Sociodemográfica de la Muestra

\begin{tabular}{|c|c|c|c|c|c|}
\hline Caso & Sexo & Edad & Tratamiento & $\begin{array}{l}\text { Sexo del } \\
\text { Terapeuta }\end{array}$ & $\begin{array}{l}\text { Duración del } \\
\text { tratamiento }\end{array}$ \\
\hline $\mathrm{E} 1$ & M & 6 & En proceso & $\mathrm{F}$ & 12 Meses* \\
\hline E2 & $\mathrm{F}$ & 11 & En proceso & $\mathrm{F}$ & 1 Mes* $^{*}$ \\
\hline E3 & $\mathrm{F}$ & 8 & En proceso & $\mathrm{F}$ & 18 Meses* \\
\hline E4 & M & 10 & En proceso & $\mathrm{F}$ & 4 Meses* \\
\hline E5 & M & 7 & Finalizado & M & $21 / 2$ Meses \\
\hline E6 & M & 7 & Finalizado & $\mathrm{F}$ & 2 Meses \\
\hline E7 & M & 10 & Finalizado & $\mathrm{F}$ & 12 Meses \\
\hline E8 & M & 7 & En proceso & $\mathrm{F}$ & 12 Meses* \\
\hline E9 & M & 11 & Finalizado & M & 12 Meses \\
\hline E10 & M & 9 & Finalizado & $\mathrm{F}$ & 2 Meses \\
\hline
\end{tabular}

Nota: M: Masculino, F: Femenino, $\left(^{*}\right)$ Al momento de la entrevista.

Fuente: elaboración propia.

\section{Materiales}

El instrumento utilizado fue una entrevista semidirigida desarrollada especialmente para esta investigación. Utilizando como base una entrevista para adultos (Olivera, Braun, Gómez Penedo y Roussos, 2013) se adaptaron los contenidos y la formulación de las preguntas para satisfacer los objetivos de la presente investigación. Se realizó una prueba piloto de la adaptación inicial y se modificaron algunos aspectos, en función de lo encontrado. La misma consistió en una entrevista a un niño que fue analizada por los autores y a partir de la misma se consensuó cuales preguntas debían agregarse o removerse. En el protocolo final se incluyeron preguntas que buscaban indagar las impresiones y percepciones de los niños sobre el tratamiento realizado, áreas relacionadas al motivo de consulta, los cambios percibidos, el vínculo con el terapeuta, las características físicas del entorno psicoterapéutico y las actividades que allí se realizaban. Durante la administración de las entrevistas se buscó que los participantes expresaran libremente sus pensamientos y sensaciones sobre la terapia realizada.

\section{Procedimientos}

El siguiente trabajo implicó la participación voluntaria de los sujetos incluidos en la muestra. Para esto se les informó sobre las intenciones del trabajo y la posibilidad de retirarse en cualquier momento que lo desearan. Una vez obtenido el asentimiento por parte de los niños, para garantizar su protección, se les entregó un consentimiento informado a los padres o tutores de los niños con la información pertinente y se les pidió que lo firmaran en caso de estar de acuerdo con su participación, teniendo siempre en cuenta el asentimiento del niño. También se tuvo en cuenta la garantía de la confidencialidad de los datos de los participantes. A cada participante se le asignó un número y aquellos datos que pudieran revelar información del participante fueron codificados.

Todas las entrevistas fueron administradas en los hogares de los niños. Una vez concluidas las preguntas de los protocolos, se realizó la toma de los datos sociodemográficos de los participantes, a partir de entrevistas a los padres, que incluían: edad, género, nivel educativo y lugar de residencia. 
En un primer momento se establecía rapport con el niño, es decir, se conversaba con el niño sobre temas de interés personal para que se sintiera cómodo y se pudiera generar un buen vínculo. Luego se realizaban las preguntas del protocolo, cuyas respuestas fueron audio-grabadas digitalmente y presentaron una duración promedio de 10 minutos ( $M=10 \mathrm{~min} ; D E=2.3 \mathrm{~min})$. Estas fueron transcritas para su posterior análisis.

Una vez finalizada esta primera instancia, se llevó a cabo el análisis de los datos, con una metodología cualitativa basada en el consenso a partir de la construcción de dominios, ideas nucleares y categorías, basadas en el modelo de Hill (2012), a fin de este estudio se modificó el dispositivo y se describe a continuación. Para empezar, se desarrollaron y codificaron los dominios. Esto implicó sintetizar porciones de material analizado en áreas temáticas. Una vez que los dominios se mostraban a lo largo de las distintas entrevistas, se los daban por adecuados. Posteriormente se redactaron las ideas nucleares, para lo cual se resumieron los dichos de los participantes en frases más simples, respetando el significado explícito de cada participante. Luego, se construyeron las categorías, lo cual implicó un análisis cruzado de todas las entrevistas. Este proceso significó agrupar ideas nucleares similares de los distintos casos y otorgarles una categoría en común. En el caso del presente trabajo, inicialmente una autora analizó las entrevistas y fue auditada por otra autora de forma independiente, con lo cual estableció un consenso que resultó en las listas finales de dominios, ideas nucleares y categorías.

Finalmente se realizó un conteo de la frecuencia de aparición de las categorías en la muestra. Según lo propuesto por Hill, Knox, Thompson, Nutt Williams y Hess (2005) se otorgaron etiquetas a las frecuencias de tal manera que: las ideas expresadas por todos los participantes o todos menos unos se consideran generales; las expresadas por más de la mitad de la muestra y hasta el punto de corte de las generales son típicas; las expresadas por más de 2 participantes, pero menos de la mitad de la muestra son variantes. Aquellas ideas expresadas por un solo participante se identifican como "atípicas" y se excluyeron de la presentación de resultados.

\section{Resultados}

Las preguntas diseñadas para recabar información en niños fueron concentradas en seis grandes dominios; 1) valoración de la terapia, 2) noción de la terapia, 3) características de la terapia, 4) cambio, 5 ) relación terapéutica y 6 ) intervenciones.

A lo largo de este apartado se exhiben citas textuales de los participantes. En ellas, se respetó cómo cada niño expresó y formuló su respuesta, tanto en el vocabulario elegido como en la construcción de las frases.

Las categorías se seleccionaron según su frecuencia de repetición. Cabe destacar que las categorías no fueron consideradas excluyentes, esto implicó que la idea expresada por cada participante puede encontrarse en más de un dominio y en más de una categoría. Y que los participantes podían tener distintas respuestas que pertenecen a distintas categorías de un mismo dominio.

Es importante señalar que el hecho de que una categoría no aparezca en un caso no implica que el paciente no pudiese estar de acuerdo con la misma. Si no que, al tratarse de entrevistas con carácter semiestructurado, ciertas cuestiones pueden no haber surgido espontáneamente durante estas.

A diferencia de lo observado en entrevistas con adultos (ver por ejemplo Olivera, 2016) los niños por lo general respondían de manera breve a las preguntas del entrevistador, y en algunas ocasiones no podían justificar coherentemente sus respuestas. Sin embargo, se consideran un importante aporte a la comprensión del modo en que experimentaron sus tratamientos.

\section{Dominio Cambio}

Dentro de las entrevistas se realizaron preguntas específicas haciendo referencia al cambio percibido por los niños. Como, por ejemplo: “¿Pensás que te ayudó en algo? ¿En qué pensás que te ayudo? ¿Notas algo diferente desde que vas? ¿En dónde / Como lo notas?". 
La primera cuestión por destacar de las respuestas obtenidas en este dominio es que la mayoría de los niños expresó alguna mejora a causa de la psicoterapia. Esto implica que ocho de los diez niños se vieron conformes con algún aspecto que haya surgido a partir del tratamiento y sólo dos expresaron que la terapia no los ayudó en ninguna cuestión que pudieran mencionar.

Por otra parte, se encontró con una frecuencia variante que uno de los cambios que los niños más resaltaban estaba relacionado a su estado anímico. Describiendo que su terapeuta les aconsejaba, y escuchaba, particularmente, con cuestiones emocionales, como la tristeza.

“ $<E>$ : ¿Vos pensás que te sirvió, que aprendiste algo?

$\langle\mathrm{P}>$ : Si, a que, si vos te aguantás la tristeza te sentís mal, y si la liberás te sentís mejor (E7)"

Se encontraron también respuestas que por su poca frecuencia se consideraron categorías únicas en donde los niños valoraron negativamente la asistencia al tratamiento, explicando que ir a terapia los aburría (en un caso), o que ir a terapia no les gustaba (en solo un caso también).

\section{Dominio Valoración de la terapia}

Una de las cuestiones valoradas por parte de los niños, era el cambio. Dentro de este dominio encontramos dos categorías de valoración en relación con el mismo, ambas con una frecuencia típica.

Por una parte, la categoría "El niño piensa que ir a terapia ayuda", incluía respuestas relacionadas a que el proceso terapéutico implicaba una ayuda o mejora. Por otro lado, la categoría "El niño atribuye características positivas a la terapia relacionadas al cambio" en donde, de forma más específica, los niños destacaban las distintas áreas donde percibían cambios.

También se encontraron dos categorías relacionadas a las intervenciones. La primera, de frecuencia típica, "El niño atribuye características positivas a las actividades de la terapia", incluía todas aquellas cuestiones que los niños destacaban que le gustaban realizar en cada sesión, como dibujar, hablar, compartir el tiempo con su terapeuta. La segunda, "Lo que más le gustaba al niño era jugar en terapia" de frecuencia variante.

Por último, nos encontramos con una categoría variante, "El niño volvería a ir a terapia". Esta respuesta surgió a partir de una pregunta tanto a los niños que no habían finalizado el tratamiento, como aquellos que sí, que era si volverían a terapia. A lo cual muchos respondieron que si tuvieran algún problema, miedo, o preocupación volverían a consultar. Al mismo tiempo esto se relaciona con el próximo dominio a analizar: "Relación terapéutica". Ya que al menos tres de los niños, destacaron que, de volver a terapia, lo harían con el mismo terapeuta y hasta llegaron afirmar que volverían a terapia tan solo para visitar a su terapeuta. En contrapartida a esto, tan solo dos niños afirmaron que no volverían a terapia, y sus respuestas se consideraron categorías únicas.

“ $<\mathrm{E}>$ : ¿Y si aparece otro problema, volverías $a$ ir a verla?

$<\mathrm{P}>$ : Si

$<\mathrm{E}>$ : ¿O irías a otra persona?

$<P>$ : A *Jaz (E3)".

Como puede apreciarse, las cuestiones a las que los niños dieron valor incluyen tanto el cambio, como las actividades que se realizan en el proceso terapéutico y el vínculo con su terapeuta. Al analizar estos resultados nos encontramos con la posibilidad de englobar y cruzar la información obtenida en los distintos dominios y en las distintas entrevistas. Dándole de esta forma un orden de jerarquía, pero sin olvidar que todos los aspectos aquí indagados están relacionados.

\section{Dominio Relación Terapéutica}

Respecto al dominio de la relación terapéutica se indagó en la valoración del niño tanto en las características del vínculo terapéutico como en las del terapeuta. Este dominio fue junto al de "Intervenciones", donde más categorías se encontraron. 
En cuanto a las características del vínculo, la primera categoría típica fue "Había confianza entre el niño y el terapeuta", que incluía las ideas en las que se expresaba confianza, donde destacaban poder contar sus cosas al terapeuta, otorgando un valor apreciativo a la relación.

“ $<E>$ ¿ ¿Y que tiene *Jaz de especial que hace que le puedas contar las cosas?

$$
\begin{aligned}
& <T>\text { : Confía en mi } \\
& <E>\text { : Confía en vos } \\
& <P>\text { : } Y \text { yo confío en ella (E3)" }
\end{aligned}
$$

Otra particularidad que los niños destacaban del vínculo era la diversión. La categoría "El niño atribuye diversión al vínculo" incluía estas ideas y tuvo una frecuencia variante.

Las últimas dos categorías relacionadas a la apreciación del vínculo fueron "El niño quiere a su terapeuta", y "El niño atribuye características positivas al vínculo". La primera, con una frecuencia variante, fue el resultado de la pregunta ¿Querés a tu terapeuta? A lo cual al menos cuatro niños respondieron que sí. La segunda, con una frecuencia típica, incluyó todas aquellas cuestiones de forma global que los niños destacaban del vínculo terapéutico y que clasificaban como agradable.

Con respecto a cómo los niños describían a su terapeuta encontramos una categoría relacionada a la descripción física, "El/La terapeuta es un adulto". Cuando se les preguntaba a los niños cómo eran sus terapeutas, resaltaban la diferencia de edad con una frecuencia típica. Por otro lado, tenemos la categoría típica "El niño atribuye al terapeuta características positivas", donde se incluían aquellas particularidades del terapeuta que los niños recalcaban.

Por último, en este dominio, encontramos dos categorías en las que se destacaron las actividades en conjunto con el terapeuta como significativas para la relación. La primera, con una frecuencia típica, "El niño le contaba sus cosas al terapeuta”, incluyó los casos donde existía facilidad por parte del niño para comunicar al terapeuta las preocupaciones y la forma en que el profesional actuaba sobre esto.
Pero también, destacaron el poder jugar con su terapeuta. De esta idea surge la categoría "El niño juega con el terapeuta", de frecuencia variante en la que los niños no solo destacaban el juego como una de las actividades más apreciadas, sino también el hecho de compartirlo con el terapeuta.

Como puede observarse a lo largo del dominio relación terapéutica, los niños expresaron empatía respecto a sus terapeutas. No solo les atribuyeron características positivas, sino que pudieron expresar que se sentían cómodos con ellos, y distinguían el vínculo con el terapeuta de vínculos con otras personas de su entorno. La información aquí obtenida se relaciona con el cambio, ya que una de las cuestiones destacadas del cambio era que se relacionaba con la figura del terapeuta.

\section{Dominio Nociones de la terapia}

Dentro del dominio de noción de la terapia se incluye la percepción del paciente sobre su terapia, sus creencias sobre la terapia en general, y la percepción que el niño posee sobre su situación dentro del tratamiento. En el mismo se encontraron 5 categorías. En primer lugar, se les preguntó a los niños porque creían que la gente iba a terapia. Esto dio como resultado la primera categoría: "El niño cree que la gente va a terapia para mejorar o solucionar problemas".

“ $<E>$ : ¿Vos porque pensás que va la gente a la psicóloga?

$$
\begin{aligned}
& <\mathrm{P}>\text { : Para ayudarlos } \\
& <\mathrm{E}>\text { : ¿Con qué? } \\
& <\mathrm{P}>\text { : Por su bien, para que puedan hacer una } \\
& \text { vida, hacerla feliz (E2)". }
\end{aligned}
$$

Una cuestión a destacar es que a pesar de que muchos niños podían explicar porque la gente iba a terapia, cuando se les preguntaba el motivo por el cual ellos realizaban el tratamiento, no todos conocían el motivo.

En relación con esta cuestión, se pudieron encontrar dos resultados. El primero, se plasmó en una 
categoría de frecuencia variante de cuatro respuestas, "El niño no sabe porque va a terapia", donde se incluían todas aquellas respuestas donde los niños expresaban ir a terapia porque sus padres le decían, los llevaban, o no sabían el por qué. Por otra parte, con seis respuestas y una frecuencia típica, la categoría "El niño sabe porque va a terapia", incluyó distintas respuestas las cuales referían a mejorar ciertas conductas, aliviar ciertos problemas o lograr bienestar en algún área. Esto puede relacionarse directamente con una tercera categoría que es "El niño atribuye a su terapia aprendizaje y mejoras". Es decir, que la noción de que la terapia esta vinculada a la mejoría, no solo cuando se habla de que terceros realicen un tratamiento, sino también cuando se refieren a sí mismos.

“<E>: ¿Y vos porque pensás que vas?

$<\mathrm{P}>$ : Para por ejemplo si vos estás enojado con tu mama, eh, vas y le decís porque estás enojado y te sentís mejor (E7)".

“ $<\mathrm{E}>$ : ¿Y por qué pensás que vas a ver a ${ }^{*} \mathrm{Clau}$ ?

$<\mathrm{P}>$ : Porque tenemos que platicar, porque a veces nos sale mal algo y tenemos que hacerlo bien" (E1).

En relación con esta creencia de que la terapia tiene una relación con el aprendizaje y el bienestar, surge otra idea de gran importancia que es el rol que los niños le adjudican al terapeuta respecto a esto. Los individuos entrevistados concedían a los profesionales ciertas características que los diferenciaban de otras personas, y que les daban la capacidad de poder ayudar. Este tipo de ideas pueden verse en el siguiente ejemplo:

“ $<E>$ : ¿Y por qué pensás que la gente va al psicólogo?

$<\mathrm{P}>$ : Para arreglar sus problemas, si estas nervioso, si hay algo que no te gusta

$<E>$ : ¿Y por qué un psicólogo y no otra persona?

$<\mathrm{P}>$ : Porque los psicólogos estudian cómo hacer eso (E10)"

\section{Dominio Características de la terapia}

En cuanto al dominio características de la terapia, el mismo se enfoca en el setting, es decir, la descripción del lugar, características físicas, modalidad de trabajo (la dinámica tanto individual como vincular). Aquí se encontraron categorías relacionadas al lugar donde se llevó a cabo la terapia. Los niños describieron que "La terapia es en un consultorio", como categoría típica, y siete de los niños explicaron que era un departamento o una casa. Al preguntarse por el sitio, los niños le adjudicaban una evaluación positiva. Otra de las características que mencionaban del mismo era "En terapia hay juguetes", lo cual fue agrupado en una categoría con frecuencia variante. Muchos de los juguetes mencionados eran juegos de mesa.

En relación con la frecuencia con la que asistían a terapia, la información fue obtenida mediante los padres. Todos aclararon que la frecuencia fue semanal en un principio, y en algunos casos fue pactándose cada dos semanas. En cuanto a la dinámica del tratamiento nos encontramos con que, de manera típica, ocho de los niños aludieron entrar solos a sesión. Esto fue categorizado como "El niño entra solo a terapia". Con una menor frecuencia, de tres referencias, algunos niños mencionaron que los padres también visitaban al terapeuta. Estas ideas se agruparon bajo la categoría "Los padres del niño ven al terapeuta”. La última categoría a resaltar en este dominio es que los niños que mencionaban que era su madre quien los llevaba a terapia. El nombre designado a esta categoría fue "La mamá del niño es quien lo lleva a terapia".

\section{Dominio Intervenciones}

En este último dominio se concentra la participación del paciente, aquellas actividades que se realizan dentro del marco del tratamiento y cuales destaca el paciente. Aquí nos encontramos con categorías que recorren las principales modalidades de trabajo mencionadas por los niños.

Para comenzar, encontramos el dibujo en distintas categorías. De forma típica con seis respuestas, los niños mencionaron dibujar en terapia, 
lo cual se agrupó en la categoría "El niño dibuja en terapia”. Pero también remarcaron una diferencia entre aquellos que dibujaban lo que querían y los que dibujaban lo que su terapeuta les decía. Se encontró una categoría de frecuencia variante "El niño no dibuja en terapia”. La misma incluyó tres respuestas que indicaban que el niño no dibujaba durante las sesiones.

Por otro lado, nos encontramos con el juego, donde se pudieron agrupar ideas en dos categorías. La primera, con una frecuencia típica, "El niño juega en terapia”, se compuso por aquellas respuestas en donde el niño mencionaba jugar en las sesiones. La segunda, "El niño lleva juegos a terapia”, incluyó aquellas respuestas que implicaban llevar a sesión juegos, u objetos que el niño quisiera compartir con su terapeuta.

Por último, encontramos categorías relacionadas a la comunicación verbal. La primera, "El niño habla en terapia”, de frecuencia típica incluyó aquellas respuestas donde el niño destacaba el diálogo.

" $<E>$ : ¿Y cuando ibas a verla a Lili* le contabas las cosas?

$<\mathrm{P}>$ : Si

$<\mathrm{E}>$ : ¿Qué le contabas?

$<\mathrm{P}>$ : Lo que hacía en el día, le contaba cosas que no me gustaban

$<E>$ ¿Y qué te decía?

$<P>$ : No me acuerdo, en una época, le conté que cuando jugaba en la casa de mi papá dejaba las cosas ahí para después seguir y él me las guardaba, y yo las quería dejar como estaban para seguir jugando, y ella me dijo que podíamos decir que eso era una pausa y era como que no podía tocar nada" (E10).

Con respecto a la participación del terapeuta en la comunicación verbal, nos encontramos con dos categorías. La primera, de frecuencia variante, "el niño y el terapeuta hablan en terapia", en donde los niños especificaban que se entablaba un diálogo en terapia. La segunda, "el terapeuta da consejos al niño", de frecuencia típica. En esta segunda, los niños marcaban intervenciones más directivas por parte del terapeuta, que llamaban consejos. Como puede observarse en el fragmento anterior y en el siguiente ejemplo:

" $<E>$ : ¿Y qué te dice *Jaz que hace que no te pelees tanto?

$<\mathrm{P}>$ : Me da un consejo

$<\mathrm{E}>$ : ¿Te acordás de alguno?

$\langle\mathrm{P}\rangle$ : Me dijo que me tenía que contener si me peleo con una amiga y después le pido perdón “(E3).

Una última cuestión a destacar es que al preguntarse a los niños sobre la existencia de tareas para realizar entre sesión de forma típica respondieron no tener tareas. Por lo cual nos encontramos con una categoría llamada "El terapeuta no da tareas entre las sesiones”, que incluyó esta idea.

Como puede observarse, las categorías del dominio pueden agruparse en tres grandes formas de intervención: el dibujo, el juego, y la comunicación verbal. A lo largo del recorrido de los anteriores dominios, estas modalidades de trabajo fueron mencionadas tanto por su valoración, como por su relación con el vínculo terapéutico.

\section{Discusión}

El presente trabajo indagó acerca de la percepción del proceso psicoterapéutico en niños. En términos generales, los resultados indican que los niños son capaces de percibir cambios y vincularlos al proceso psicoterapéutico. Los distintos participantes pudieron manifestar algún crecimiento o resolución de las problemáticas trabajadas en el espacio de psicoterapia. Los cambios percibidos abarcaron cuestiones tanto conductuales, como interpersonales y emocionales, entre otras. Respecto a esto, la categoría con mayor frecuencia se relacionó con la mejora en el estado anímico. Lo cual coincide con los resultados de la línea de investigación de Midgley y colaboradores (2018), donde los pacientes adultos entrevistados destacaban el poder manejar 
sus sentimientos como consecuencia de su paso por la terapia cuando eran niños. El cambio es un aspecto valorado positivamente por los niños, algunas de las cuestiones que parecían agradables de ir a terapia se relacionaban con que implicaba una ayuda, una forma de solucionar problemas o aprendizaje.

Al igual que lo observado en el estudio de Kazdin y Durbin (2012), en esta investigación aquellos niños que percibieron un motivo para asistir al tratamiento mantuvieron una actitud más participativa con relación a las tareas y la comprensión de la psicoterapia. Por el contrario, aquellos que expresaban no ir por voluntad propia, a la hora de definir su actitud con relación al cambio y a la terapia lo hacían de forma negativa.

Cabe destacar que además del cambio percibido y las actitudes de los niños los participantes señalaron la importancia del rol del terapeuta a la hora de motorizar el cambio. Por ejemplo, cuando se les preguntaba a los niños por su terapeuta, estos no sólo los caracterizaban de forma positiva, si no que afirmaban que existía una diferencia entre su terapeuta y "otras personas". Una de las cualidades básicas que resaltaban era que sus terapeutas eran adultos. Una posible lectura de esta caracterización es que a pesar de que los terapeutas jugaban con los niños y estos los describían como divertidos, se resaltaba una diferencia en la edad, demostrando que los niños no conciben al terapeuta como un par. Otra conclusión respecto a la relación con el profesional fue que aquellos niños que destacaban positivamente al terapeuta eran los que mayores cambios atribuían a la terapia. Estos resultados se encuentran en consonancia con investigaciones cualitativas realizadas con adultos (Olivera et al., 2013)

Con relación a la concepción de alianza terapéutica (Bordin, 1979), pudo observarse como mencionan Zack y colaboradores (2007) que los niños presentan una falta de metas respecto al tratamiento. Cuando se les preguntaba a los niños el porqué de los cambios o de realizar un tratamiento psicoterapéutico, tenían dificultad en explicar la relación entre estas cuestiones. Los mismos eran capaces de entender que la terapia servía para "mejorar", pero no encontraban la explicación sobre cómo era que la terapia los ayudaba. Aun más, algunos niños pudieron identificar algunas razones que tienen las personas para acceder a una psicoterapia, pero no de explicar por qué ellos mismos lo hacían. Lo cual nos lleva a preguntarnos si las metas, tal como las podemos pensar, forman parte de un contexto del adulto y, en el caso de los niños, no tienen necesariamente la misma condición en el tratamiento.

Respecto a las intervenciones utilizadas, observamos coincidencias con los estudios mencionados en el estado del arte y el marco teórico del trabajo (Drake y Winner, 2012; Landreth, 2012; Malchiodi, 2003). Se encontró que el juego fue la intervención más destacada por los participantes, haciendo énfasis en que era una actividad compartida con su terapeuta y que era "divertido". Esto puede relacionarse con la idea postulada por Landreth (2012) que sostiene que el juego es un medio natural de comunicación en los infantes, como lo es la verbalización en los adultos. El juego apareció como una actividad de intercambio, en donde los niños se sentían cómodos. A pesar de que los niños no hicieron una conexión concreta entre el juego y el cambio, calificaron al juego como una actividad propia de la terapia y describieron mejoras o actitudes más funcionales como resultado de su ejercicio. Además, al destacar aquello que más les gustaba de la terapia, el juego aparecía con frecuencia, también atribuyendo diversión al vínculo terapéutico.

Por otra parte, respecto al dibujo encontramos que los niños expresaron dibujar en terapia. Malchiodi (2003) menciona que esta actividad puede verse direccionada en distintos grados por el terapeuta. Teniendo en cuenta los resultados obtenidos, encontramos que la misma cantidad de niños expresaron dibujar lo que ellos querían y lo que su terapeuta les decía. Por lo tanto, se concluye que el dibujo era utilizado en distintas modalidades. Adicionalmente se observó que aquellos niños que mencionaron dibujar en terapia también mencionaron mejoras en su estado anímico. Esta cuestión concuerda con la investigación realizada por Drake y Winner (2012). Estos resultados nos permiten conocer que los niños discriminan las distintas actividades, pero no sabemos hasta qué punto se les explica o no estratégicamente el para qué de las mismas a lo largo del tratamiento. 
Finalmente, teniendo en cuenta que el cambio es percibido y asociado con el proceso de la psicoterapia, sería interesante continuar trabajando si es posible pensar un esquema de metas que quede al servicio de las tareas del proceso psicoterapéutico.

\section{Limitaciones y futuras investigaciones}

La presente investigación, cuenta con una serie de limitaciones a destacar. Como es propio de las investigaciones cualitativas, los resultados obtenidos no tienen como objetivo poder generalizarse, sino comprender fenómenos desde distintas perspectivas abarcando su complejidad (Vasilachis de Gialdino, 2006). El bajo número de casos no permite la generalización de los resultados, pero muestra una tendencia que tendría que ser luego corroborada en investigaciones cuantitativas y experimentales. Dentro de las limitaciones, también se debe tener en cuenta que, a excepción de un caso, todos los niños concurrían a terapia en un ámbito privado, es decir, el lugar del tratamiento era en un consultorio privado. Esto implica que no se brinda información sobre otros tratamientos, por lo cual sería interesante a futuro realizar investigaciones con muestras más amplias y diversas respecto a esta característica incluyendo, por ejemplo, niños que realicen tratamientos en hospitales públicos.

Como se ha mencionado anteriormente, existen escasas investigaciones en relación al cambio en psicoterapia en niños y a la perspectiva de los mismos. Conocer las experiencias de los pacientes brinda la oportunidad a los psicoterapeutas de reflexionar sobre la práctica clínica, para así contribuir a la efectividad de la misma. Resultaría interesante a futuro trabajar con esta población indagando sobre las características del vínculo terapéutico y los factores mediadores que llevan al cambio. También sería importante como mencionan Schmidt y Schimmelmann (2015) realizar estudios que reconozcan a los tres informantes involucrados en la psicoterapia en niños (terapeuta, padres y niño).

A modo de cierre se puede concluir que cada uno de los elementos se vincularon entre sí conformando un entramado, y no se conciben como elementos aislados del proceso psicoterapéutico. Los niños tuvieron la posibilidad de expresar sus creencias respecto a la psicoterapia, las cuestiones que más valoraban de la misma, su vínculo con el terapeuta, las actividades que realizaron en el tratamiento, y los resultados del mismo.

\section{Referencias}

Bordin, E. (1979). The Generalizability of psychoanalitic concept of the working alliance. Psychoterapy: Theory reseach and practice. 16, 252-260. https://doi.org/10.1037/h0085885

Bunge E., Gomar M. y Mandil, J. (2009), Terapia cognitiva con niños y adolescentes: Aportes Técnicos - 2da ed. Buenos Aires: Akadia.

Cantoni Rabolini, N. M. (2009) Técnicas de muestreo y determinación del tamaño de la muestra en investigación cuantitativa. Revista Argentina de Humanidades y Ciencias Sociales, 7(2).

Castaño, H. D., Posada, I. C. y Agudelo, O. E. (2019). La importancia de escuchar a los jóvenes. Diversitas: Perspectivas en Psicología, 15(1), 27-36. https://doi.org/10.15332/ s1794-9998.2019.0001.02

Diamond, S. y Lev-Wiesel, R. (2016). The title "therapy" and what do you do with it as a child? Recollections of being in child expressive arts group therapy. Clinical Child Psychology and Psychiatry, 22(1),152-164. https://doi. org/10.1177/1359104516656723

Drake, J. E. y Winner, E. (2012): How children use drawing to regulate their emotions. Cognition \& Emotion, 27(3), 512-520. https://doi.org/10. 1080/02699931.2012.720567

Fernández Álvarez, H. (2011) El campo de la psicoterapia. En H. Fernández Álvarez (Ed) Paisajes de la psicoterapia. Buenos Aires: Polemos.

Hill, C. E., Knox, Thompson, B. J., Nutt Williams, E., y Hess, S. A. (2005). Consensual Qualitative Research: An Update. Journal of Counseling Psychology, 52(2), 196-205. https://doi. org/10.1037/0022-0167.52.2.196 
Hill, C. (2012) Consensual qualitative research : a practical resource for investigating social science phenomena. Washington, DC: American Psychological Association.

Kazdin, A. E. y Durbin, K.A. (2012). Predictors of child-therapist Alliance in cognitive-behavioral tratment of children referred for oppositional and antisocial behavior. Psychotherapy, 49, 202-217. https://doi.org/10.1037/a0027933

Landreth, G. L. (2012). Play therapy: The art of the relationship. New York, NY: Routledge.

Laurenceau, J.-P., Hayes, A. M. y Feldman, G. C. (2007). Some methodological and statistical issues in the study of change processes in psychotherapy. Clinical Psychology Review, 27(6), 682-95. https://doi.org/10.1016/j. cpr.2007.01.007

Malchiodi, C. (2003). Handbook of art therapy. New York, NY: Guildford Publications, Inc.

Midgley, N., Target, M. y Smith, J. (2006). The outcome of child psychoanalysis from the patient's point of view: a qualitative analysis of a long-term follow-up study. Psychology and psychotherapy, 79(2), 257-269. https://doi. org/10.1348/147608305X52694

Midgley, N., Capella, C., Goodman, G., Lis, A., Noom, Ma., Tishby, O. y Weitkamp, K. (2018). Introduction to the special section on child and adolescent psychotherapy research. Psychotherapy Research, 28, 1-2. https://doi. org/10.1080/10503307.2017.1380864

Molina, M. F., Giménez, M., Raimundi, M. J., Leibovich de Figuero, N. y Schmidt, V. (2017). ¿Cómo perciben los adolescentes de la ciudad de Buenos Aires su futuro? Una mirada desde sus actores. Diversitas: Perspectivas en psicología, 13(2), 241-253. http://dx.doi.org/10.15332/ s1794-9998.2017.0002.08
Olivera, J., Braun, M., Gómez Penedo, J. M. y Roussos, A. (2013). A qualitative investigation of former clients' perception of change, reasons for consultation, therapeutic relationship, and termination. Psychotherapy, 50(4), 505-516. https://doi.org/10.1037/a0033359

Olivera, J. (2016) Nociones de cambio en psicoterapia desde la perspectiva de sujetos que han realizado un tratamiento psicoterapéutico. [Tesis doctoral]. Facultad de Psicología. Universidad de Buenos Aires.

Roussos, A. (2013). Introduction to special section on clients' perspective of change in psychotherapy. Psychotherapy, 50(4), 503-504. https://doi.org/10.1037/a0033847

Schmidt S. J. y Schimmelmann B.G. (2015). Mechanisms of change in psychotherapy for children and adolescents: current state, clinical implications, and methodological and conceptual recommendations for mediation analysis. European Child \& Adolescent Psychiatry, 24, 249-253 https://doi.org/10.1007/ s00787-015-0698-0

Sempik, J., Ward, H. y Darker, I. (2008). Emotional and behavioural difficulties of children and young people at entry into care. Clinical Child Psychology and Psychiatry, 13(2), 221-233. https://doi.org/10.1177/1359104507088344

Stern M. (2002). Child Friendly Therapy. Biopsychosocial innovations for children and families. New York: W.W Norton \& Company.

Vasilachis de Gialdino, I. (2006). Estrategias de investigación cualitativa. Barcelona: Gedisa.

Zack, S. E., Castonguay, L. G. y Boswell, J. F. (2007). Youth working alliance: A core clinical construct in need of empirical maturity. Harvard Review of Psychiatry, 15, 278-288. https://doi. org/10.1080/10673220701803867 